\title{
Assessing sprinkler systems performance with a novel experimental benchmark
}

\author{
Andrea Petroselli, ${ }^{1}$ Dario Romerio, ${ }^{2}$ Piero Santelli, ${ }^{2}$ Roberto Mariotti, ${ }^{3}$ Silvano Di Giacinti, ${ }^{3}$ \\ Luca Casini, ${ }^{1}$ Carmine Testa ${ }^{4}$
}

${ }^{1}$ Department of Economics, Engineering, Society and Business Organization (DEIM), Tuscia University, Viterbo;

${ }^{2}$ Irritrol Systems Europe Srl, Fiano Romano (RM); ${ }^{3}$ ARSIAL, Agenzia Regionale per lo Sviluppo e l'Innovazione

dell'Agricoltura del Lazio, Azienda Sperimentale di Tarquinia, Tarquinia (VT); ${ }^{4}$ Department of Agriculture and

Forestry (DAFNE), Tuscia University, Viterbo, Italy

\begin{abstract}
Sprinkler systems are one of the most popular methods of irrigation worldwide. One of their key parameters is the so-called level of uniformity, i.e. every portion of the soil should be irrigated with the same amount of water. Assessing the level of uniformity is crucial for optimal design of sprinkler systems. In this manuscript, a novel experimental benchmark is presented in order to test irrigation sprinklers, assess their performance, and define their acceptable working conditions. Different sprinklers have been tested, their water application depth curves have been determined, and their performance has been evaluated using a combination of metrics. Results show that the majority of sprinklers are characterized by very good performance in terms of operating pressures in the range 2.0-3.0 bar and tend to decrease their efficiency for operating pressures outside of that range.
\end{abstract}

\section{Introduction}

An irrigation process with impact sprinklers consists of a high-velocity water jet dispersed into the air in a set of droplets, that are distributed over the ground surface with the aim of achieving a uniform distribution (Tarjuelo, 1999). Sprinkler systems are one of the most popular methods of irrigation worldwide, covering more than $50 \%$ of the total irrigated land (USDA, 2009). Their use

Correspondence: Andrea Petroselli, Department of Economics, Engineering, Society and Business (DEIM), Tuscia University, 01100 Viterbo, Italy. E-mail: petro@unitus.it

Key words: Experimental benchmark; irrigation; sprinkler; level of uniformity.

Received for publication: 26 March 2021.

Accepted for publication: 25 May 2021.

${ }^{(C)}$ Copyright: the Author(s), 2021

Licensee PAGEPress, Italy

Journal of Agricultural Engineering 2021; LII:1172

doi:10.4081/jae.2021.1172

This article is distributed under the terms of the Creative Commons Attribution Noncommercial License (by-nc 4.0) which permits any noncommercial use, distribution, and reproduction in any medium, provided the original author(s) and source are credited. is increasing up to $90 \%$ in several countries, like France (Saretta et al., 2018). In the field, sprinklers can be arranged in a variety of regular and irregular shapes and patterns to irrigate many different crops (Santelli, 2016).

One of the key parameters in designing an irrigation plant is the so-called level of uniformity, i.e. every portion of the soil should be irrigated with the same amount of water. If this circumstance is achieved, yield and plant quality will usually be maximized (Zhang et al., 2013). Obviously, a 100\% level of uniformity is not possible due to a number of reasons, from the type of weather conditions (e.g. wind speed and direction) to topographic characteristics (land elevation and pipeline path alter water pressure and hence the artificial rainfall intensity that is proportional to the pressure head inside pipelines). Aside from environmental factors, another source of uncertainty in achieving irrigation uniformity is the sprinkler itself, and in particular its structural characteristics. Indeed, in theory, the water jet sprayed from the sprinkler at a high velocity and dispersing into the air in droplets should be adequately uniform, but the sprinkler physical features, its nozzle configuration and operating water pressure strongly modify the amount of water reaching the ground, causing losses in the level of uniformity (Amer, 2006). It is noteworthy that the performance of sprinkler systems can be assessed also using other parameters or indexes, like the relationship between flow rate and operating pressure, or the coefficient of pulverization (Li and Kawano, 1998).

Performance assessment of sprinkler systems is usually based on a water distribution chart, which is essentially a plot of the application rate measured (using simple collectors) along the jet length with various statistical uniformity coefficients, which were proposed over the past decades (Al-Ghobari 2006). For instance, Maroufpoor et al. (2010) reported the following uniformity coefficients: Christiansen's (1942), Wilcox and Swailes (1947), Criddle et al. (1956), Benami and Hore (1964), Hart and Reynolds (1965), Beale and Howell (1966), Karmeli (1978), Merriam and Keller (1978), Hawaiian Cane Society Specialists (Merriam and Keller, 1978).

The assessment of the level of uniformity and other characteristics of the investigated sprinkler system is always a complex and time-consuming task. The procedure usually consists in arranging a certain number of collectors (catch-cans) on the ground in a rectangular or radial layout with respect to the sprinkler, according standardized methods (e.g. ISO7749-1 and ISO15886-3; ISO, 1995, 2012). Then, a series of tests (often in an indoor laboratory, in order to exclude the wind effect and other environmental influences) are repeated, measuring the water collected in the single catch-can. Such tests have rigorous, long, and repetitive procedures, and the manual nature of the work is also labour demanding. Because of this, for many commercial sprinklers only concise information is often provided (like the average rainfall intensity). 
Therefore, practitioners who have to design an irrigation system are lacking detailed information for assessing their performance at the desired operating pressure. As to irrigation uniformity, sprinkler systems arranged in the selected pattern on the soil require a minimum value for the level of uniformity. Low values are usually indicative of a faulty combination of the number and size of nozzles, operating pressure and/or mutual spacing or overlap of sprinklers.

The objectives of this work are two. Firstly, we present a recently developed experimental benchmark for testing irrigation sprinklers that can help identifying their typical water distribution curves. Secondly, we assess the performance of the investigated sprinklers and define their acceptable working conditions, using a combination of selected metrics.

\section{Materials and methods}

\section{The experimental benchmark}

The experimental benchmark was developed at the testing facility of ARSIAL (Lazio Region Agency for Development and Innovation in Agriculture) near Tarquinia, Central Italy in a greenhouse (internal dimensions $24 \times 12 \mathrm{~m}$ ), in order to exclude the wind effect. The experimental benchmark is dynamic and modular, and can be adapted to the characteristics of the investigated sprinklers, as explained in the following. The experimental benchmark is characterized by an inner octagon with each side of approximately $0.4 \mathrm{~m}$ which makes it possible to install up to eight rows of modular slim rectangular panels added in series. Each modular panel ( $4.5 \mathrm{~m}$ long and $0.25 \mathrm{~m}$ wide) is equipped with small rain gauges (top diameter $75 \mathrm{~mm}$ ), numbered progressively and distant $0.3 \mathrm{~m}$ from each other, for a total of 15 rain gauges for each panel. The modular panels can be positioned in sequence starting from each side of the octagon to obtain different lengths to investigate according to the desired setup. The investigated sprinkler is located at the centre of the octagon. Sprinkler and rain gauges are located at the same elevation (simulating ground level at zero slope) thanks to a series of elevation regulators to adapt to the soil surface. Depending on the characteristics of the investigated sprinkler (such as the jet throw and/or the spray angle) different layouts can be arranged. Figure 1 reports an example of a layout for the experimental benchmark is presented, while Figure 2 shows some details.

\section{The hydraulic layout}

The water feeding the system can come from a groundwater source and/or pressurized pipeline owned by the local authority. The water coming from any of these sources is filtered by an automatic double chamber sand filter ( 75 micron) and a safety disc filter (115 micron). An additional $1 \mathrm{~m}^{3}$ tank in proximity of the greenhouse with an external $1.1 \mathrm{~kW}$ pump (Calpeda MGPM 405) can be used, if the circuits need to be disconnected. An electronic programmer with a closing ball valve is able to operate the circuit, ensuring the desired discharge. A pressure head controller (OMR 100) after the valve regulates the hydraulic load from 1 to 6 bar. The system also includes a digital flowmeter, an analogue thermometer and a digital manometer. The water is then routed to the sprinkler at the centre of the inner octagon thanks to a flexible plastic pipeline. A second analogue manometer is installed in the proximity of the sprinkler. A bucket with a pulley system for jet inhibition during test pressure calibration can be operated manually to cover the investigated sprinkler. The bucket (cfr. Figure 2) can be lifted up or lowered instantly to start and end each test at the same time. This avoids that rainfall is collected in the rain gauges due to the sprinkler initial unsteady flow or during the pressure regulation operations. Ancillary data, such as air temperature, air humidity, barometric pressure, wind speed and velocity are measured using a movable weather station that was set within the greenhouse. Some details of the hydraulic layout are shown in Figure 3.

\section{The performed analysis}

The modular panels are arranged starting from the inner octagon depending on the jet throw and the spray angle of the investigated sprinkler. For sprinklers with $45^{\circ} / 90^{\circ} / 180^{\circ} / 270^{\circ} / 360^{\circ}$ spray angles, 1/1/3/4/5 rows of modular panels are used, respectively, according Figure 4.

It is noteworthy that, due to the inner greenhouse dimensions,

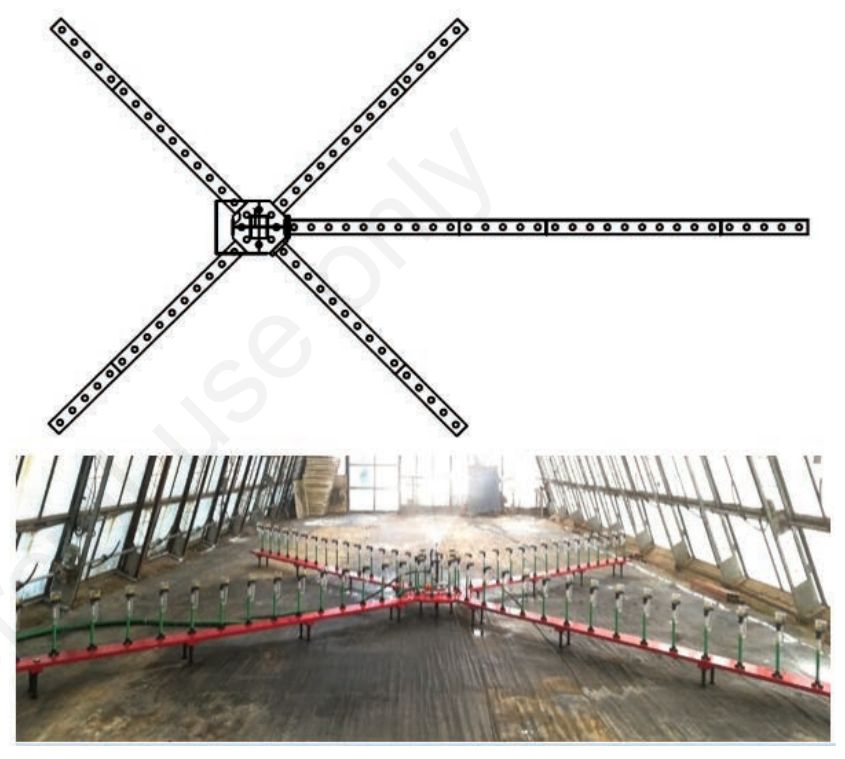

Figure 1. The experimental benchmark. Schematic representation of inner octagon and rows of modular slim rectangular panels (up). Example of experimental benchmark in action (down).

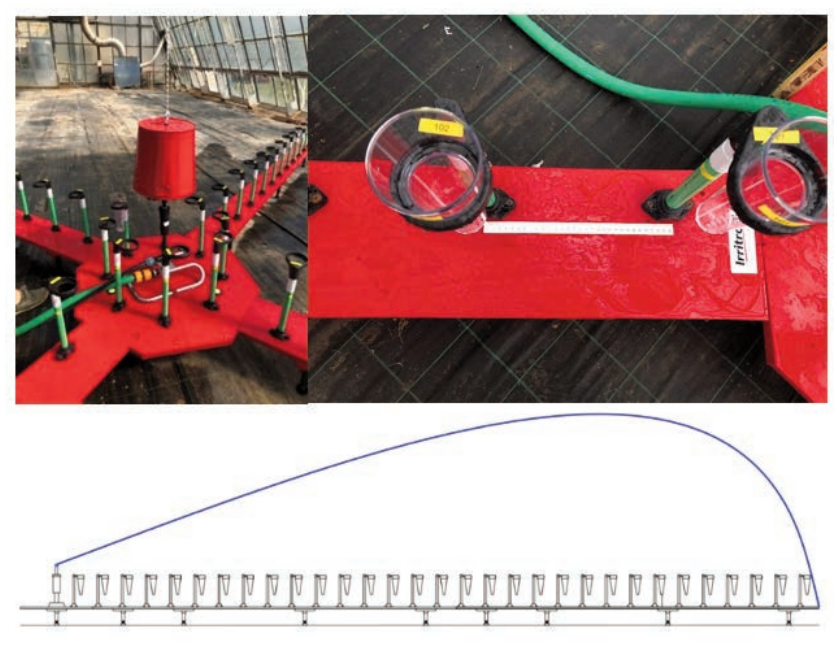

Figure 2. Details of the experimental benchmark: Inner octagon (top, left); two rain gauges on modular panel (top, right); scheme of two modular panels in series set on one side of the octagon (bottom) for testing a sprinkler with a jet throw equal to $9.3 \mathrm{~m}$. 
in case of $180^{\circ} / 270^{\circ} / 360^{\circ}$ spray angle sprinklers, the maximum jet throw that can be investigated is approximately $5 \mathrm{~m}$, since the inner octagon is positioned in the greenhouse centre. Conversely, in case of $45^{\circ} / 90^{\circ}$ spray angle sprinklers, a maximum jet throw of $23 \mathrm{~m}$ can be investigated, shifting the inner octagon on the edge of the greenhouse, as shown in Figure 5.

After having positioned the modular panels, based on the selected sprinkler, a single test starts with the opening of the valve which controls the water flow. At the beginning of the test, the bucket covers the sprinkler, and it is lifted up only after that the pressure has been stabilized at the desired value. After the bucket lifting, a digital chronometer starts recording the test duration, that lasts until the moment when the first rain gauges reach a water level approximately half of their height, in order to measure a suitable water volume. At the end of the test, the bucket is lowered, the chronometer stops to determine the test end and its duration, and the water circuit is closed. All the rain gauges are then weighed using an electronic precision balance, and the weight of the rain gauge, previously measured, is subtracted. Then, the water volume can be determined based on water density which is known (depending on the recorded water temperature). Finally, the water volume is divided by the rain gauge surface area and the test duration to calculate the sprinkler rainfall intensity for the whole investigated ground surface, which lead to the determination of the sprinkler application rate.

Knowing the sprinkler application rate, it is possible to assess the sprinkler performance. For this purpose, we selected the following metrics in this paper:

i) The Christiansen's coefficient (Christiansen, 1942):

$$
C U=100\left(1-\frac{\sum_{i=1}^{n}\left|X_{i}-\mu\right|}{\sum_{i=1}^{n} X_{i}}\right)
$$

where $C U$ is the Christiansen's coefficient (\%), $\mathrm{n}$ is the number of the depth measurements of the water applied, $\mathrm{X}_{\mathrm{i}}$ is the measured application depth (L), and $\mu$ is the mean application depth (L).

ii) The distribution uniformity in the lower quartile (Merriam and Keller, 1978):

$$
D U=100 \frac{h_{q}}{\mu}
$$

where $D U$ is the distribution uniformity in the lower quartile (\%), and $\mathrm{h}_{\mathrm{q}}$ is the mean application depth in the less irrigated quartile (L).

iii) The scheduling coefficient (Burt et al., 1997):

$$
S C=\frac{\mu}{h_{\text {crit }}}
$$

where $S C$ is the scheduling coefficient (-), and $\mathrm{h}_{\text {crit }}$ is the mean application depth (L) in the unit length. In the calculation of SC, we used a $2.5 \%$ value of the relative size of the critical dry area. Indeed, in literature values in the range $2 \%-10 \%$ are commonly used (see for instance ISO. 2012. ISO15886-3).

These three metrics are directly related to the level of unifor-

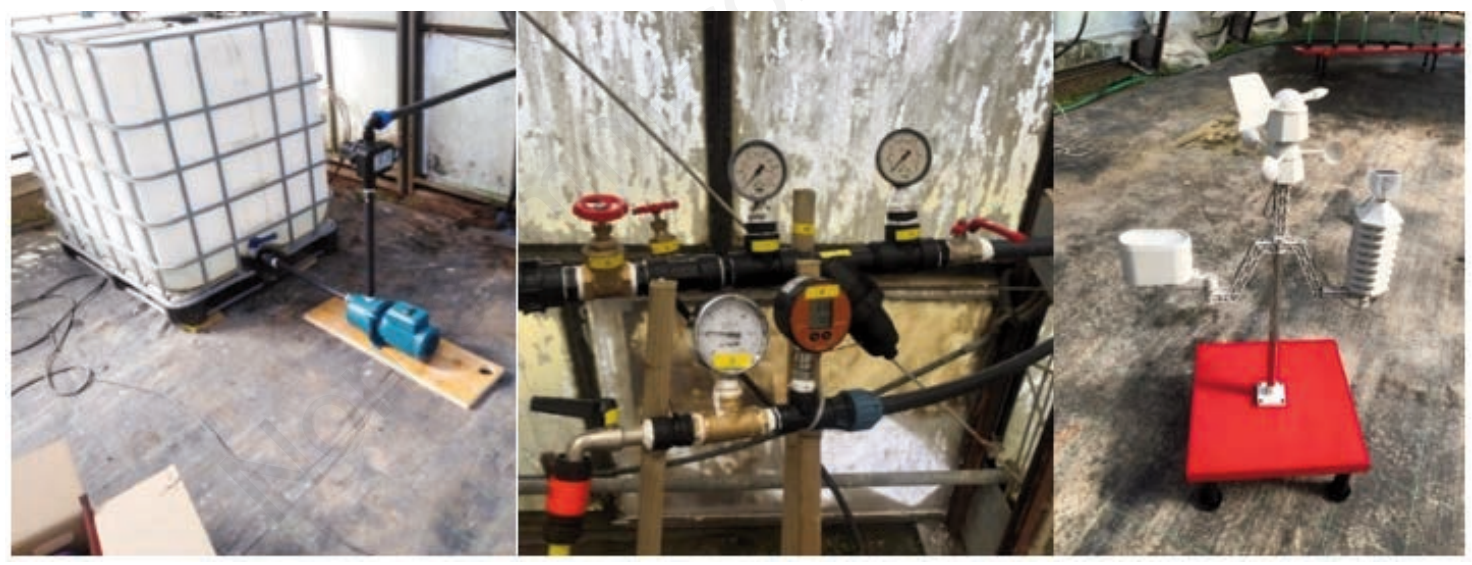

Figure 3. Details of the hydraulic layout: the $1 \mathrm{~m}^{3}$ tank and external pump (left), regulation system and instruments (centre), weather station (right).

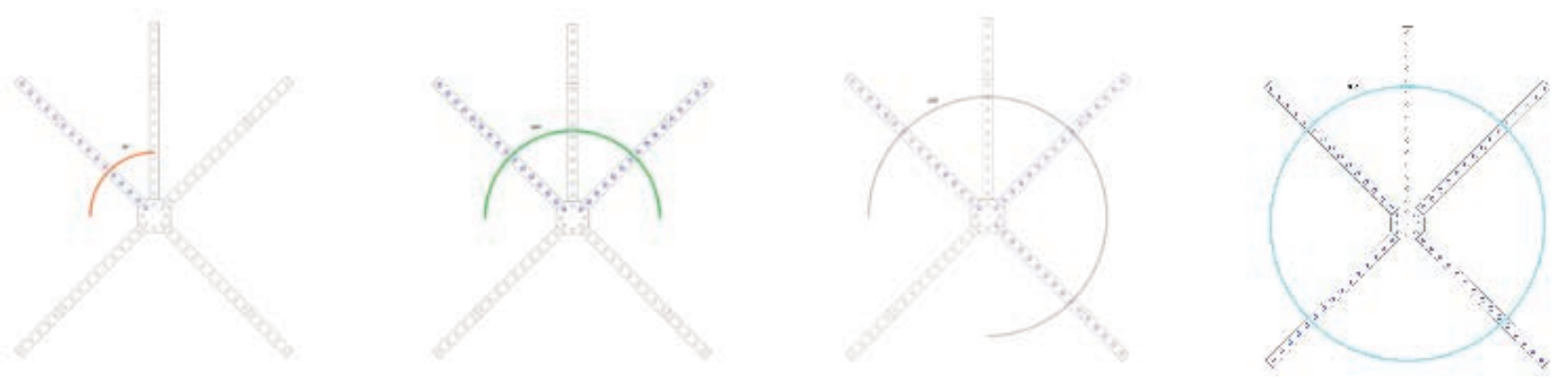

Figure 4. Example of arrangement of modular panels depending on spray angle $\left(45^{\circ}-90^{\circ} ; 180^{\circ} ; 270^{\circ} ; 360^{\circ}\right.$ from left to right). 
mity, because they measure how every portion of the soil is irrigated compared with the others. It is noteworthy that the sprinklers are usually arranged on the ground following different patterns to maximize the level of uniformity thanks to a selected overlap. Indeed, the overlap makes it possible to minimize the area where the application depth is minimal, and also to compensate for the wind effect. The geometrical shape method (triangular or rectangular pattern) is probably the simplest and most widely used spacing method, because it provides the highest uniformity for the system. Hereafter we make reference to CU, DU and SC values obtained thanks to that particular overlap which maximises them, as this circumstance represents one of the most used system for arranging sprinklers on the ground (Saretta et al., 2018). As shown in the literature, an irrigation system requires a minimum value of such metrics to be considered as acceptable. For instance, regarding CU, the irrigation uniformity can be classified as 'not acceptable' when $\mathrm{CU}$ is below $70 \%$, 'scarce' for $70 \%<\mathrm{CU}<74.9 \%$, 'sufficient' for $75 \%<\mathrm{CU}<79.9 \%$, 'good' for $80 \%<\mathrm{CU}<82.9 \%$, 'very good' for $83 \%<\mathrm{CU}<87 \%$, 'excellent' for $\mathrm{CU}>87$ (Keller and Bliesner, 1990). In order to assess the selected sprinklers based on all the aforementioned metrics simultaneously, we did not consider their absolute values, but we assigned relative ranks to the investigated metrics following the criteria reported in Table 1. In particular, the classification of Table 1 is based on the works of Merriam and Keller (1973), Keller and Bliesner (1990), Zoldoske et al. (1994) and Islam et al. (2017). For each of the considered metric, the investigated sprinkler can achieve a rank between 0 (lowest performance) and 5 points (highest performance), based on the collected application depths that in turn are a function of a certain operating pressure. Therefore, it is possible to determine the relationship among the overall rank for the sprinkler (that can yield a maximum of 15 points in which a greater value represents better sprinkler performance) and the operating pressure, thus defining its best working conditions. It is noteworthy that the previously mentioned metrics depend on the spacing between the catch-cans, that in this case were placed at a distance of $0.3 \mathrm{~m}$ from each other. In conclusion, the procedure for assessing the investigated metrics and the sprinklers' optimal overlap and working conditions can be summarized as follows. First, we measured the water collected in the catch-cans at a distance of $0.3 \mathrm{~m}$ from each other. This operation was performed for each individual sprinkler, without any overlap. Second, we used Microsoft Excel to simulate different distances between two facing sprinklers with a spatial discretization of $0.3 \mathrm{~m}$ to calculate virtually the water application depth and the investigated metrics. Third, we calculated the overall rank of the sprinkler for all the overlapping distances. Forth, we selected that particular overlapping distance providing the greatest value for the overall rank and determined the corresponding values for CU, DU and SC. It is noteworthy that the calculations regarding the overlap were performed only considering the line joining two sprinklers, without evaluating typical layouts used commonly in agriculture, such as triangular and rectangular layout.

\section{The investigated sprinklers}

The sprinklers investigated in this manuscript were supplied by
Irritol and belong to the Irritrol range NEW I-PRO-FTM (NIPF). In detail, we tested the NIPF-15Q, NIPF-15H, NIPF-15TQ and NIPF-15F, where Q means a jet angle of $90^{\circ}, \mathrm{H}$ means a jet angle of $180^{\circ}$, TQ means a jet angle of $270^{\circ}$, and $\mathrm{F}$ means a jet angle of $360^{\circ}$. For each of the considered sprinklers, we tested operating pressures of $1.5,2.0,2.5,3.0,3.5$ bar. All the investigated sprinklers have a nominal maximum jet throw lower than 5 meters, so we used only one modular panel for each line, reaching a maximum distance for the rain gauges of 4.8 meters from the sprinkler. As explained above, we used one line for the $90^{\circ}$ spray angle tests (for a total of 16 rain gauges), three lines (each one at each side of the octagon, for a total of 48 rain gauges) for the $180^{\circ}$ spray angle tests, four lines (each one at each side of the octagon, for a total of 64 rain gauges) for the $270^{\circ}$ spray angle tests, and five lines (each one at each side of the octagon, for a total of 80 rain gauges) for the $360^{\circ}$ spray angle tests. Each test was repeated two times for each configuration, and the application depths pertaining to each rain gauge in the two repetitions were averaged.

\section{Results and discussion}

As explained above, all the tests on the investigated sprinklers were performed inside a greenhouse, in order to exclude the wind effect and create a sort of reference condition useful for designers. It must to be noted, indeed, that the same experimental tests, conducted outdoor, could have been strongly different, mainly due to

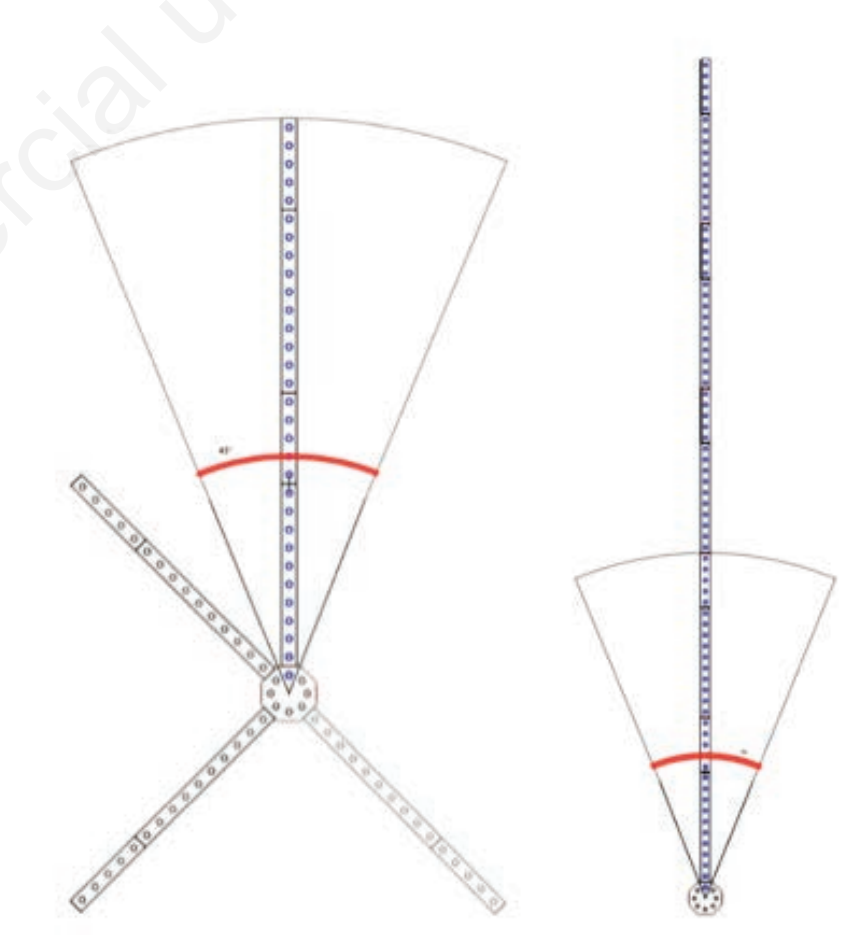

Figure 5. Example of arrangement of modular panels depending on jet throw.

Table 1. Ranks for the selected metrics for the uniformity assessment (calculated indoor and with a spacing of catch-cans equal to $0.3 \mathrm{~m}$ ).

\begin{tabular}{lcccccc} 
& Not acceptable & Poor & Fair & Good & \multicolumn{2}{c}{ Very good Excellent } \\
CU (\%) & $<70$ & $70-74.9$ & $75-79.9$ & $80-82.9$ & $83-87$ & $>87$ \\
DU (\%) & $<65$ & $65-69.9$ & $70-74.9$ & $75-79.9$ & $80-85$ & $>85$ \\
\hline SC (-) & $>1.5$ & $1.5-1.41$ & $1.4-1.31$ & $1.3-1.21$ & $1.2-1.11$ & $<1.1$ \\
Rank (points) & 0 & 1 & 2 & 3 & 4 & 5 \\
\hline
\end{tabular}


the wind effect and evaporation, that could strongly alter the amount of rainfall collected in the catch-cans. However, we believe that our results can give useful suggestions for the selection of one investigated sprinkler instead of another, and for the evaluation of its performance.

Environmental parameters were within the following intervals: $54 \%-96 \%$ for air humidity, $16^{\circ} \mathrm{C}-29^{\circ} \mathrm{C}$ for air temperature, $16^{\circ} \mathrm{C}$ $22^{\circ} \mathrm{C}$ for water temperature. Since the reported intervals are quite large, we proceeded to a double weighting in order to exclude the effect of water evaporation from the rain gauges during weighting, which in the case of sprinklers working at a $360^{\circ}$ spray angle, could last for some time due to the high number of rain gauges to process. This was done in particular with high values of air temperature. However, the double weighting showed no significant differences in terms of application depth inside the individual rain gauges.

In Figure 6, we show the water distribution curves as a function of the distance from the sprinkler centre, without considering the sprinkler overlap done in the field. In Figure 6, we have just one sprinkler positioned at the origin of the axis. The results depicted in Figure 6, as explained above, were obtained by averaging the application depths observed in the rain gauges at the same
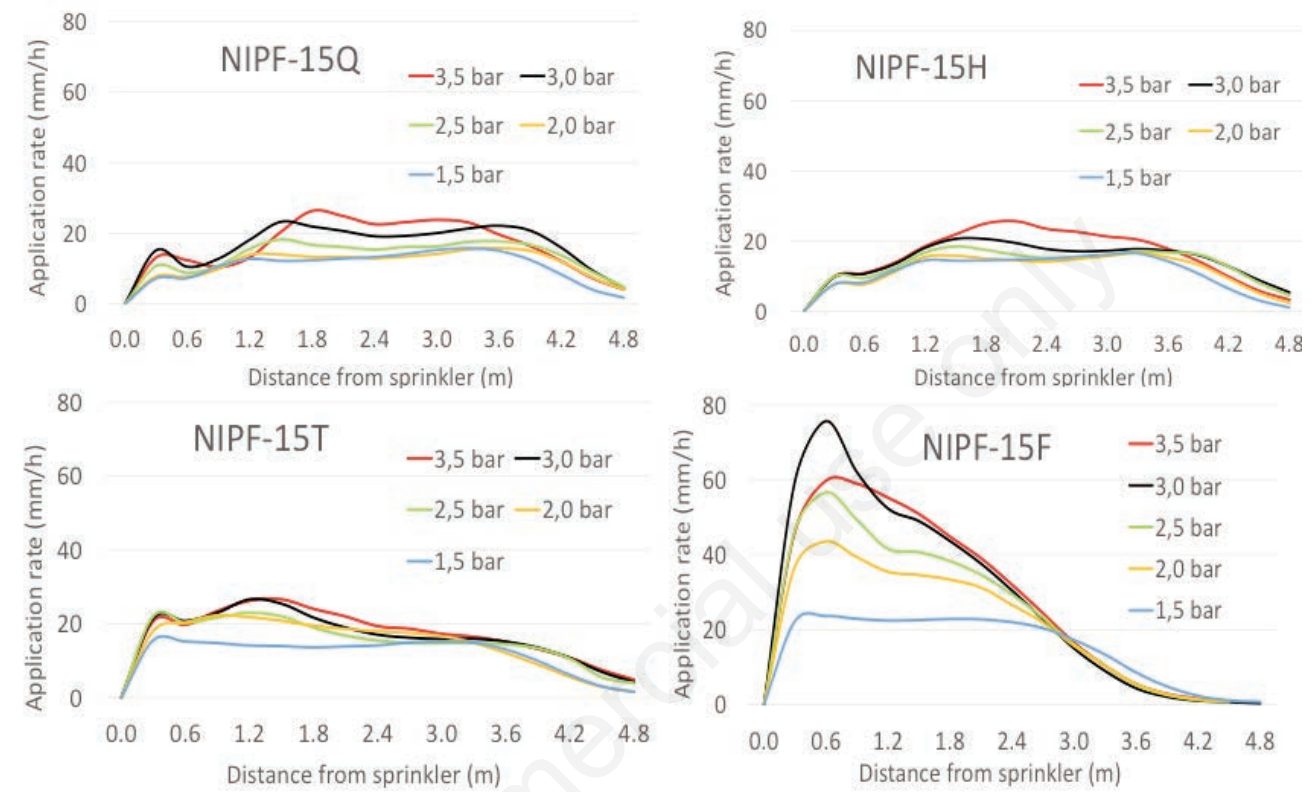

Figure 6. Water distribution curves (without overlap). The sprinkler is located at the origin of the axis.
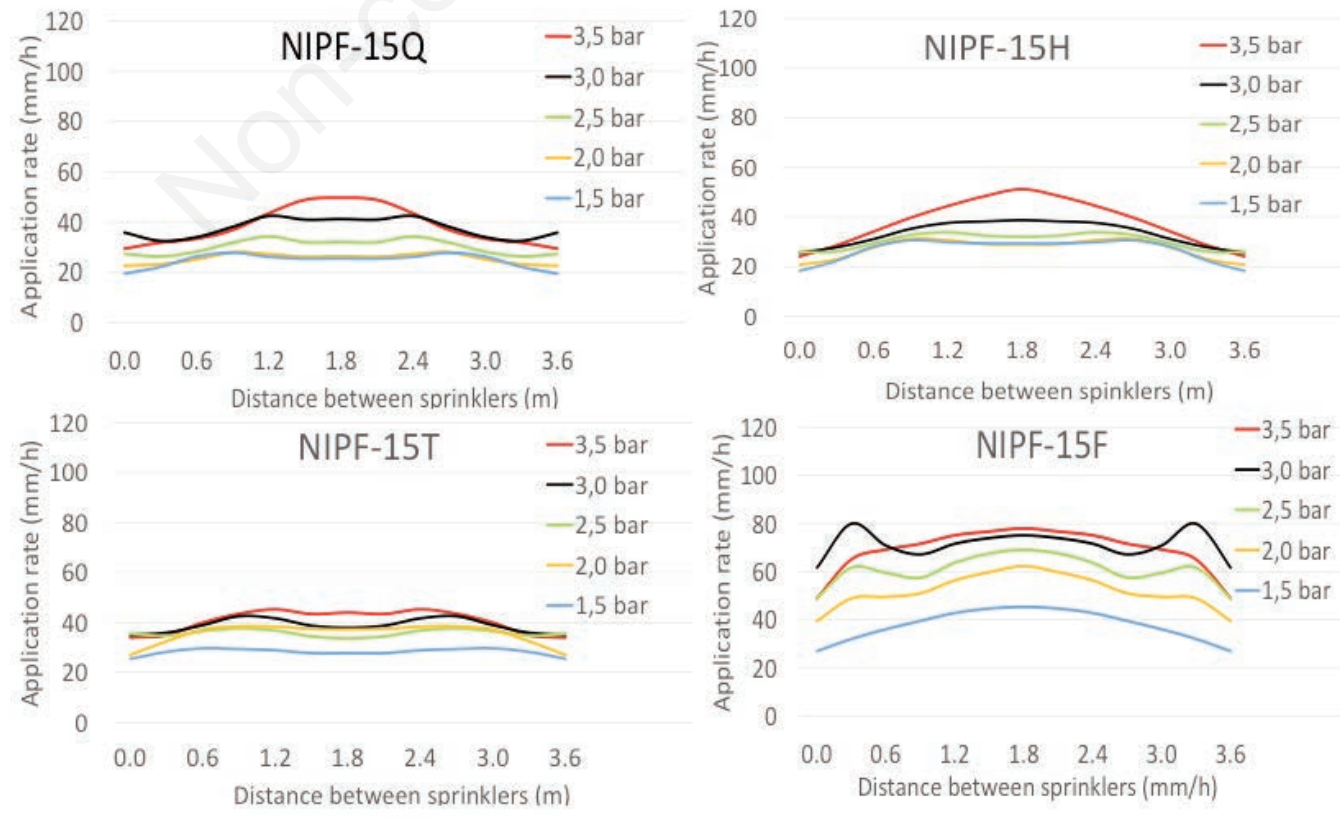

Figure 7. Water distribution curves (with overlap). Two sprinklers are facing each other and are positioned at the extremes of the application rate curves. 
distance from the sprinkler centre and pertaining to different lines. Each application depth in the rain gauges is the average of two repetitions on each test. As can be seen from Figure 6, the water distribution curves have an expected irregular shape, with the water concentrated in proximity of the sprinkler, followed by a decreasing trend. Such irregular behaviour is more pronounced for NIPF$15 \mathrm{~F}$, while the other sprinklers are characterized by smoother water application curves. It is noteworthy that an increasing pressure does not always produce an increment in the jet throw, as expected from a theoretical point of view. For NIPF-15F, operating pressures greater than 1.5 bar produce a slight decrease in the jet throw, probably due to the high level of pulverization. This creates smaller and lighter water particles, which cannot reach large distances from the sprinkler. The obtained results are congruent with literature findings (e.g. Zanon et al., 2000; Amer, 2006; Do Prado, 2016). In particular Topak et al. (2005) recommended that the sprinkler systems should operate between 2.0 and 3.5 bar to achieve a good irrigation uniformity.

In Figure 7, we show the water distribution curves considering the particular sprinkler overlap maximizing the total rank provided by the investigated metrics for assessing the sprinkler performance and discussed previously. In Figure 7, we have two sprinklers facing each other at the extremes of the water application rate curves. As can be seen from Figures 6 and 7, and as expected, the effect of the overlap on the application depth is evident. The overlap allows to obtain a much better level of uniformity that can be quantified using the investigated metrics previously described. It is noteworthy that for the selected sprinklers the overlap decreases the distance between two consecutive sprinklers, which, in order to reach the maximum uniformity level, should be placed at a distance of

\begin{tabular}{|lccccc|}
\hline Model)Pressure (bar) & $\mathbf{1 . 5}$ & $\mathbf{2 . 0}$ & $\mathbf{2 . 5}$ & $\mathbf{3 . 0}$ & $\mathbf{3 . 5}$ \\
\hline NIPF-15F & & & & & \\
CU (\%) & 85.4 & 89.3 & 92.1 & $\mathbf{9 4 . 0}$ & 89.7 \\
DU (\%) & 78.4 & 85.4 & 88.0 & $\mathbf{9 0 . 5}$ & 83.2 \\
SC (-) & 1.19 & 1.13 & 1.14 & $\mathbf{1 . 1 0}$ & 1.20 \\
Rank (points, max = 15) & 11 & 14 & 14 & 14 & 12 \\
\hline NIPF-15T & & & & & \\
CU (\%) & 92.3 & 88.0 & 94.6 & 92.4 & 89.6 \\
DU (\%) & 95.2 & 84.3 & 96.2 & 91.6 & 85.1 \\
SC (-) & 1.16 & 1.27 & $\mathbf{1 . 0 5}$ & 1.10 & 1.21 \\
Rank (points, max =15) & 14 & 13 & 15 & 15 & 14 \\
\hline NIPF-15H & & & & & \\
CU (\%) & 79.4 & 83.2 & $\mathbf{8 8 . 0}$ & 84.1 & 74.2 \\
DU (\%) & 76.3 & 80.3 & $\mathbf{8 6 . 1}$ & 80.7 & 69.9 \\
SC (-) & 1.56 & 1.41 & $\mathbf{1 . 1 6}$ & 1.23 & 1.43 \\
Rank (points, max =15) & 9 & 12 & 14 & 12 & 5 \\
\hline NIPF-15Q & & & & & \\
CU (\%) & 84.4 & 90.2 & 88.8 & 84.6 & 77.7 \\
DU (\%) & 84.6 & 90.0 & $\mathbf{8 9 . 1}$ & 83.9 & 80.2 \\
SC (-) & 1.38 & 1.21 & $\mathbf{1 . 1 5}$ & 1.15 & 1.24 \\
Rank (points, max = 15) & 13 & 14 & 14 & 12 & 11 \\
\hline LOW: Rank < 5 & & & & & \\
\hline MEDIUM: 6 < Rank < 10 & & & & & \\
\hline HIGH: Rank > 10 & & & & & \\
\hline
\end{tabular}

Figure 8. Uniformity assessment for the investigated sprinklers. The best values for each operating pressure are reported in bold. approximately $3.6 \mathrm{~m}$ compared to the jet throw that is equal to approximately $4.8 \mathrm{~m}$ for all the tested models. As to the investigated metrics for assessing the sprinkler performance, the results are summarized in Figure 8. Concerning the $\mathrm{CU}$ and considering all the operating pressures, average values are $90.1 \%$ for NIPF-15F, 91.4\% for NIPF-15T, 81.8\% for NIPF-15H, 85.1\% for NIPF-15-Q. Concerning the DU and considering all the operating pressures, average values are $85.1 \%$ for NIPF-15F, 90.5\% for NIPF-15T, $78.6 \%$ for NIPF-15H, $85.5 \%$ for NIPF-15-Q. Concerning the SC and considering all the operating pressures, average values are 1.15 for NIPF-15F, 1.16 for NIPF-15T, 1.36 for NIPF-15H, 1.23 for NIPF-15-Q. The obtained results are in line with previous findings. For instance, Tarjuelo et al. (1999) and Jobbàgy and Krystof (2018) analysed sprinklers in the range of operating pressures from 2 to 4 bar and obtained CU values in the range of 70\%-95\%.

As to the values reported in Figure 8, the following considerations can be made. NIPF-15T (jet angle at $270^{\circ}$ ) is the sprinkler which achieved the best results at all the operating pressures with an average total rank of 14.2 points out of a maximum of 15 points. This sprinkler showed an excellent performance at all operating pressures, but the best uniformity was achieved at operating pressures of 2.5 and 3.0 bar. NIPF- $15 \mathrm{~F}$ (jet angle $360^{\circ}$ ) is the second best sprinkler at all operating pressures with an average total rank of 13 points out of a maximum of 15 points. The recommended operating pressure range is between 2.0 and 3.0 bar, although the best performance is achieved at an operating pressure of 3.0 bar. NIPF-15Q (jet angle $90^{\circ}$ ) is the third best sprinkler at all operating pressures with an average total rank of 12.8 points out of a maximum of 15 points. The recommended operating pressure range is between 2.0 and 2.5 bar, with the best performance achieved at an operating pressure of 2.5 bar. NIPF-15H (jet angle $180^{\circ}$ ) turned out to be the worst-performing sprinkler at all operating pressures with an average total rank of 10.4 points out of a maximum of 15 points. It is noteworthy, anyway, that its performance rapidly decreases for lower (1.5 bar) and higher (3.5) operating pressures. However, if we consider an operating pressure in the range of 2.0-3.0 bar, its performance is good and in line with the other investigated sprinklers. It seems from our results that NIPF- $15 \mathrm{H}$ is particularly sensitive to changes in the operating pressures. Of course, the obtained results are valid only for the investigated sprinklers belonging to the NEW I-PRO-FTM line. In case of investigation on other kinds of sprinklers, the analysis should be repeated and the results could be different.

\section{Conclusions}

In this paper, a novel experimental benchmark was developed and presented in order to test irrigation sprinklers and assess their performance in order to define their suitable working conditions. Different sprinklers supplied by Irritrol and belonging to the Irritrol NEW I-PRO-FTM series were tested and their performances was evaluated using a combination of metrics. The results, indeed valid only for the investigated sprinklers, show that the majority of the NEW I-PRO-FTM sprinklers display a very good performance at operating pressures in the range of 2.0-3.0 bar, while their efficiency tends to decrease at operating pressures outside of the aforementioned range. Future investigations will have to focus on other types of sprinklers, and the development of a new and automatic open air experimental benchmark based on the prototype built indoor. The new system will make it possible to test sprinklers with a long jet throw in outdoor conditions. Indeed, at the moment, the ARSIAL greenhouse internal dimensions and the experimental benchmark layout modularity allow for testing sprinklers with a jet 
throw of only up to $10 \mathrm{~m}$, whereas longer jet throws can be tested outside the greenhouse. Moreover, the new outdoor system will allow to consider also the effect of climatic variables, such as wind and evaporation, which indeed can have a strong influence on the rainfall collected in the catch-cans.

\section{References}

Al-Ghobari H.M. 2006. Effect of maintenance on the performance of sprinkler irrigation systems and irrigation water conservation. Food Sci. Agric. Res. Center Res. Bull. 141:1-16.

Amer K.H. 2006. Water distribution uniformity as affected by sprinkler performance. Misr J. Ag. Eng. 23:66-79.

Beale J.G., Howell D.T. 1966. Relationship among sprinkler uniformity measures. J. Irrig. Drainage Engine.-ASCE. 92:41-8.

Benami A., Hore F.R. 1964. A new irrigation sprinkler distribution coefficient. Trans. ASAE 7:157-8.

Burt C.M., Clemmens A.J., Strelkoff T.S., Solomon K.H., Bliesner R.D., Hardy L.A., Howell T.A., Eisenhauer D.E. 1997. Irrigation performance measure: Efficiency and uniformity. J. Irrig. Drain. Eng. 123:423-42.

Christiansen J.E. 1942. Irrigation by sprinkling. California Agriculture Experiment Station Bulletin, No. 670.

Criddle W.D., Davis S., Pair C.H., Shockley C.D. 1956. Methods of evaluating irrigation systems. Agriculture Handbook No. 82, Soil Conservation Service, USDA, Washington, DC, USA.

Do Prado G. 2016. Water distribution from medium-size sprinkler in solid set sprinkler systems. Revista Brasileira de Engenharia Agrícola e Ambiental 20,3, 195-201.

Hart W.E., Reynolds W.N. 1965 Analytical design of sprinkler systems. Trans. ASAE 8:83-9.

Islam Z., Mangrio A., Ahmad M., Akbar G., Muhammad S., Umair M. 2017. Application and distribution of irrigation water under various sizes of center pivot sprinkler systems. Pakistan J. Agric. Res. 30:415-25.

ISO. 1995. ISO7749-1: Agricultural irrigation equipment Sotating sprinklers - Part 1: Design and operational requirements. International Standardization Organisation, Geneva, Switzerland.

ISO. 2012. ISO15886-3: Agricultural irrigation equipment Sprinklers - Part 3: Characterization of distribution and test methods. International Standardization Organisation, Geneva, Switzerland.

Jobbàgy J., Kristof K. 2018. Evaluation of the coefficient of uniformity and nonuniformity of irrigation for wide-range irrigators in various field conditions. Res. Agr. Eng. 64:55-62.
Karmeli D. 1978. Estimating sprinkler distribution pattern using linear regression. Trans. ASAE 21:682-6.

Keller J., Bliesner R.D. 1990. Sprinkle and trickle irrigation. AVI Book, Van Nostrand Reinhold, New York, NY, USA.

Li J., Kawano H. 1998. Sprinkler performance as affected by nozzle inner contraction angle. Irrig. Sci. 18:63-6.

Maroufpoor E., Faryabi A., Ghamarnia H., Moshreshreshreshrefi G.Y. 2010. Evaluation of uniformity coefficients for sprinkler irrigation systems under different field conditions in Kurdistan Province (Northwest of Iran). Soil Water Res. 5:139-45.

Merriam, J.L., Keller. J. 1973. Irrigation system evaluation and improvement. Utah State University, Logan, UT, USA.

Merriam J.L., Keller J. 1978. Farm irrigation system evaluation: a guide for management. Department of Agricultural and Irrigation Engineering, Utah State University, Logan, UT, USA.

Saretta E., de Camargo A.P., Botrel T.A., Frizzon J.A., Koech R., Molle, B. 2018. Test methods for characterising the water distribution from irrigation sprinklers: Design, evaluation and uncertainty analysis of an automated system. Biosyst. Engine. 169:42-56.

Santelli P. 2016. Metodi e tecniche di irrigazione del verde ornamentale - Architettura irrigua. Flaccovio Editore, Palermo, Italy.

Tarjuelo J.M. 1999. Irrigation uniformity with medium size sprinklers Part I: Characterization of water distribution in no-wind conditions. Trans. Am. Soc. Agric. Eng. 42:665-75.

Tarjuelo J.M., Montero J., Honrubia F.T., Ortiz J.J., Ortega J.F. 1999. Analysis of uniformity of sprinkle irrigation in a semiarid area. Agric. Water Manage. 40:315-31.

Topak R., Suheri S., Ciftci N., Acar B. 2005. Performance evaluation of sprinkler irrigation in a semi-arid area, Pakistan J. Biol. Sci. 8:97-103.

USDA. 2009. Farm and ranch irrigation survey - special studies part 1 - census of agriculture (Vol. 3). AC-07-SS-1.

Wilcox J.C., Swailes G.E. 1947 Uniformity of water distribution by some under tree orchard sprinkler. J. Sci. Agric. 27:565-83.

Zanon E.R., Testezlaf R., Matsura E.J. 2000. A data acquisition system for sprinkler uniformity testing. Appl. Engine. Agric. 16:123-7.

Zhang L., Merkley G. P., Pinthong K. 2013. Assessing whole field sprinkler irrigation application uniformity. Irrig. Sci. 31:87e105.

Zoldoske D.F., Solomo K.H., Norum E.M. 1994. Uniformity measurements for turf grass: centre for Irrigation Technology, California State University, Fresno, USA. Available from: http://cati.csufresno.edu/cit/r ese/94/941102.index.html 